\title{
Transbronchial lung cryobiopsy (TBCB) performed in acute COVID-19 pneumonia: first report
}

\begin{abstract}
A COVID-19 diagnosis is usually based on PCR detection of viral RNA in airway specimens in a patient with typical clinical features. Histological features of the COVID-19 lung disease are reported from autopsies. Transbronchial cryobiopsy (TBCB) is an evolving technique usually performed in the diagnosis of interstitial lung disease.

We report a TBCB in a 76-year-old female patient who had repeatedly tested negative for SARS-CoV-2 infection. The pathological examination revealed the presence of interstitial pneumonia with lymphocytic infiltration. The qRT-PCR against SARS-CoV-2 from a pharyngeal swab was positive after performing the TBCB.
\end{abstract}

Key words: COVID-19; pneumonia; transbronchial lung cryobiopsy

\section{Introduction}

Coronaviruses are known animal and human pathogens that have been getting more attention after the evolution of the novel coronavirus as an identified cause of a cluster of pneumonia cases in Wuhan, a city in the Hubei Province of China [1]. On March 11, 2020, the WHO classified SARS-CoV-2, the causative agent of COVID-19, as a pandemic due to the rapid spread of the disease worldwide. At the time of writing, COVID-19 had spread to 184 countries [2].

Clinical and radiological features of COVID-19 have been described in many studies [3]China, was caused by a novel betacoronavirus, the 2019 novel coronavirus (2019-nCoV. However, pathological findings from postmortem studies are still insufficient. Only a few reports have been published that address the pathological features of the COVID-19 pneumonia and ARDS [4, 5]. To our knowledge, there are no reports of small biopsies from lung tissue because COVID-19 pneumonia itself is not an indication for transbronchial lung biopsy. In this report, we describe a transbronchial lung cryobiopsy (TBCB) as well as an endobronchial ultrasound-guided transbronchial needle aspiration (EBUS-TBNA) of an enlarged mediastinal lymph node in a patient with COVID-19 lung disease.

\section{Case description}

A 76-year-old female patient was referred to our center for further management. In February 2020, the patient suffered from flu-like symptoms which were partially relieved after 10 days of treatment with Amoxicillin und clavulanic acid. However, exertional dyspnea persisted. The patient was tested twice using nasopharyngeal swabs for qRT-PCR against SARS-CoV-2 and was negative both times (PCR-Platform of the BD Max System from Becton Dickinson with the VIASURE SARS-CoV-2 S gene Real Time PCR Detection from Certest). For further diagnosis, a chest CT (performed April $6^{\text {th }}$ ) revealed bilateral peripheral pulmonary consolidations. On admission, the patient was afebrile and acyanotic. Auscultation of the lungs revealed the presence of fine inspiratory crackles bilaterally. Following a multidisciplinary discussion, a decision was made to perform a bronchoscopy, specifically a transbronchial lung 

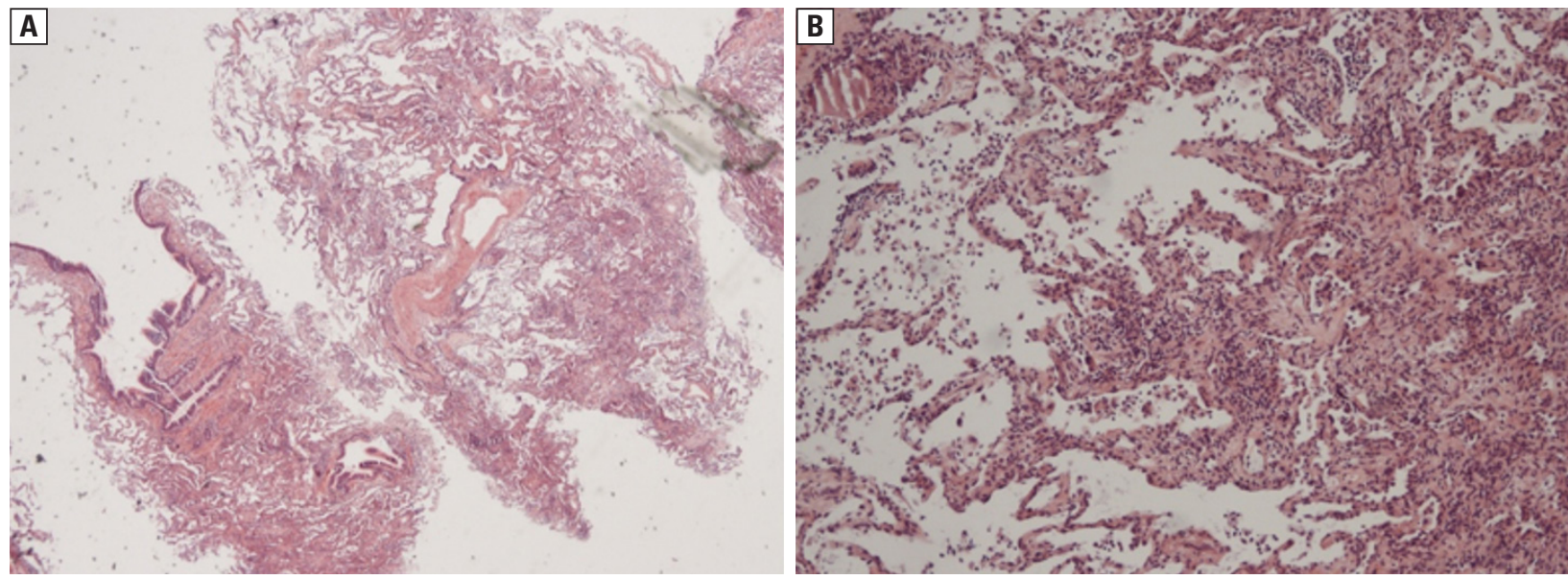

Figure 1. A. Overview with patchy peribronchial broadening of alveolar septa and interstitial inflammatory infiltrates (HE, 20×). B. A more detailed view with mixed interstitial inflammation, fibroblastic foci, and a protein precipitate (upper left) (HE, 100×)

cryobiopsy, in order to establish a diagnosis. CryoTBB was performed from the peripheral right lower lobe. Additionally, an EBUS-TBNA was performed from a subcarinal lymph node (Position 7, $10,3 \mathrm{~mm}$ ). The histopathological results showed an interstitial pneumonia caused by an infiltration with lymphocytes. Subsequently, a swab was taken from the hypopharynx and qRT-PCR against SARS-CoV-2 was repeated for the third time, this time yielding a positive result and confirming the diagnosis of COVID-19 pneumonia.

After two further weeks, the patient was discharged after two negative COVID-19 tests without residual symptoms or an indication for oxygen supplementation.

\section{Pathological findings}

The cell block from the mediastinal lymph node showed small, complex, and bruised lymphatic tissues with macrophages and anthracotic pigments. The pathological examination of the Transbronchial Lung Cryobiopsy showed significant inflammatory changes with occasionally detectable spherical protein precipitates containing foreign bodies, activated pneumocytes, and moderate mixed inflammatory infiltrate such as T-lymphocytes, macrophages, and some neutrophils containing fibroblastic buds, which were partially septal and partially intra-alveolar in location. This finding indicates the presence of interstitial pneumonia (Figure 1,2).

\section{Discussion}

The total number of confirmed COVID-19 cases exceeds 3,6 million worldwide at the time of the writing of this case report [6]. Despite that, the data about the disease, especially the histopathological data, is still growing. The lack of pathological information may be related to the sudden nature of the outbreak, the large number of affected patients that exceeds the capacity of health care facilities in many countries, and the fear of disease transmission during the invasive procedures when taking a biopsy [4].

The decision to take a biopsy from the lung was made in order to establish a diagnosis due to the suspicious ILD. This decision was made as a result of the CT imaging which showed an atypical pattern, as well as the atypical presentation of the patient (afebrile patient). Also, before the procedure, the qRT-PCR against SARS-CoV-2 was negative two times. Transbronchial lung cryobiopsy (TBCB) offers a higher diagnostic yield in parenchymal lung diseases compared to a regular transbronchial forceps biopsy due to larger sample sizes and reduced tissue artifacts [7]the resultant tissue samples are of high quality, and the lung parenchyma seen in the samples is adequate for a histological diagnosis in most cases. Bleeding after transbronchial biopsy is the most important procedure- associated complication and may be life threatening. This study addresses the risk of bleeding of transbronchial cryobiopsy. Methods: In this prospective, randomized, controlled multicentre study 359 patients with interstitial lung disease requiring diagnostic bronchoscopic tissue sampling were included. Both conventional transbronchial forceps biopsy and transbronchial cryobiopsy were undertaken in each patient. The sequence of the procedures was randomized. Bleeding severity was evaluated semi-quantitatively as \"no bleedingl", |"mildl” (suction alone. The 

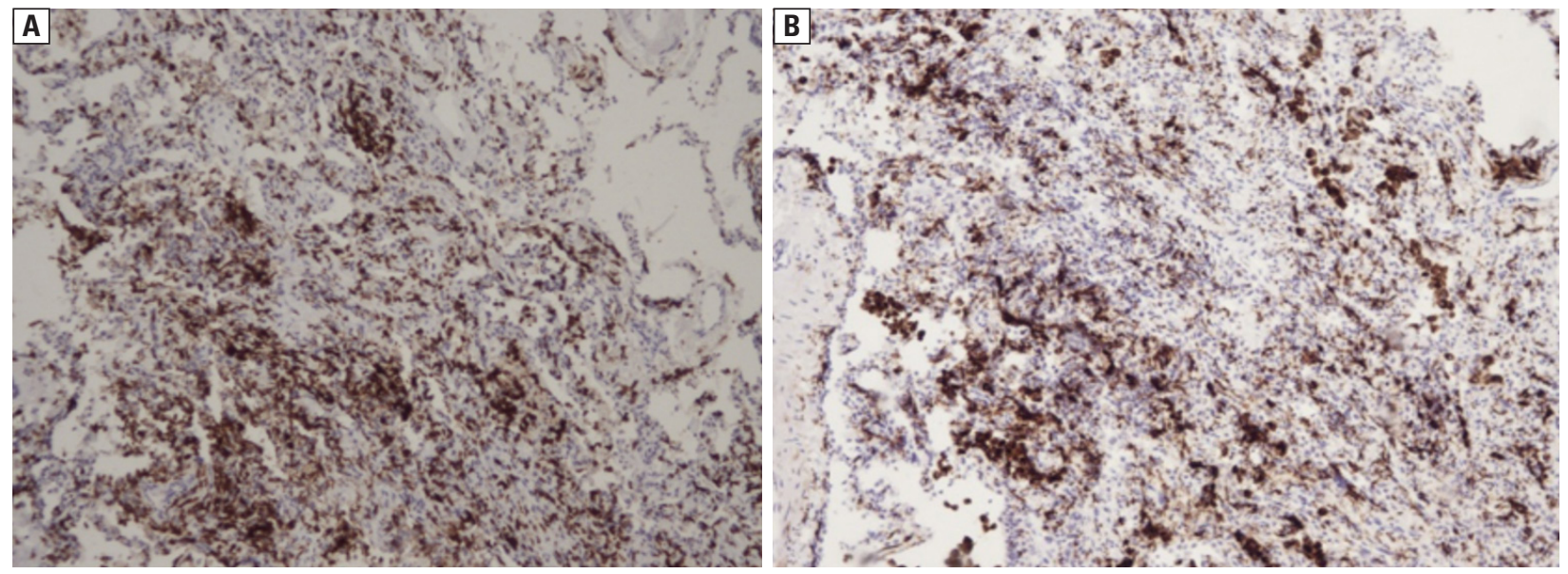

Figure 2. Immunohistological characterization of the inflammatory infiltrate with T-lymphocytes, macrophages, and nearly a complete absence of B-lymphocytes. A. Anti-CD3, 100×. B. Anti-CD68, 100×

pathological examination in our patient showed a complete picture of Acute Interstitial Pneumonia with significant lymphocytic infiltration. The patient suffered from exertional dyspnea and radiological imaging revealed bilateral consolidations.

The overall evaluation of the patient could be described as non-severe COVID-19 pneumonia. Those findings represent an early phase of the COVID-19 infection [8]. As a result of this, in our report we describe the pathological findings that could be found in early COVID-19 lung disease.

The clinical course of the COVID-19 infection can be classified as a mild form of the infection in the vast majority of the patients (80\%), severe illness in about $14 \%$ of the patients, and critical illness (including severe ARDS, sepsis, and septic shock) in about $5 \%$ of the patients [9].

The difference in the clinical presentations is explained by the difference in the pathological findings that have been described in patients with COVID-19-ARDS in which features of diffuse alveolar damage (DAD) are present. These features include hyaline membrane formation, fibrin exudates, epithelial damage, and diffuse type II pneumocyte hyperplasia with features of bacterial superinfection [4]. The fibrotic changes are rare. Available pathological data is still limited and lung biopsies from surviving patients with non-severe disease have not yet been published. The DAD features were reported in postmortem studies in patients with severe ARDS $[4,5]$.

The pathological findings in COVID-19 pneumonia were described in one report describing two patients who underwent lobectomy for adenocarcinoma and were found to have COVID-19. The pathological examination revealed the presence of edema, pretentious exudate, focal reactive hyperplasia of pneumocytes with patchy inflammatory cellular infiltration, and multinucleated giant cells. The hyaline membrane was not prominent [4].

In our report, we describe novel pathological findings of non-severe COVID-19 pneumonia obtained by the innovative technique of transbronchial lung cryobiopsy (TBCB) that contribute to the overall limited available information about the pathology of the disease.

\section{Conflict of interest}

None declared.

\section{References:}

1. World Health Organization. WHO Director-General's remarks at the media briefing on 2019-nCoV on 11 February 2020. Available online: https://www.who.int/director-general/speeches/detail/who-director-general-s-remarks-at-the-media-briefingon-2019-ncov-on-11-february-2020. [Last access: 25.02.2021].

2. Guan WJ, Ni Z, Hu Y, et al. Clinical characteristics of coronavirus disease 2019 in China. N Engl J Med. 2020; 382: 1708-1720, doi: 10.1056/NEJMoa2002032.

3. Huang C, Wang Y, Li X, et al. Clinical features of patients infected with 2019 novel coronavirus in Wuhan, China. The Lancet. 2020; 395(10223): 497-506, doi: 10.1016/s0140-6736(20)30183-5.

4. Tian S, Hu W, Niu Li, et al. Pulmonary pathology of early phase 2019 novel coronavirus (COVID-19) pneumonia in two patients with lung cancer. J Thorac Oncol. 2020; 15(5): 700-704, doi: 10.20944/preprints202002.0220.v2.

5. Hu Z, Shi L, Wang Y, et al. Pathological findings of COVID-19 associated with acute respiratory distress syndrome. Lancet. 2020; 8(4): 420-422, doi: https://doi.org/10.1016/S2213-2600(20)30076-X.

6. Worldometer. Coronavirus cases. Worldometer. pp. 1-22, 2020.

7. Hetzel J, Eberhardt R, Petermann C, et al. Bleeding risk of transbronchial cryobiopsy compared to transbronchial forceps biopsy in interstitial lung disease - a prospective, randomized, multicentre cross-over trial. Respir Res. 2019; 20(1): 140, doi: 10.1186/s12931-019-1091-1, indexed in Pubmed: 31277659.

8. World Health Organization. Clinical management of COVID-19. Available online: https://www.who.int/publications/i/item/clinical-management-of-covid-19. [Last accessed at: 25.02.2021].

9. Weekly CC. The epidemiological characteristics of an outbreak of 2019 novel coronavirus diseases (COVID-19) - China, 2020. China CDC Weekly. 2020; 2(8): 